This item was submitted to Loughborough's Research Repository by the author.

Items in Figshare are protected by copyright, with all rights reserved, unless otherwise indicated.

\title{
Pair-correlation functions and phase separation in a two-component point Yukawa fluid
}

\section{PLEASE CITE THE PUBLISHED VERSION}

http://dx.doi.org/10.1063/1.2162884

\section{PUBLISHER}

(C) American Institute of Physics

\section{VERSION}

VoR (Version of Record)

\section{PUBLISHER STATEMENT}

This work is made available according to the conditions of the Creative Commons Attribution-NonCommercialNoDerivatives 4.0 International (CC BY-NC-ND 4.0) licence. Full details of this licence are available at: https://creativecommons.org/licenses/by-nc-nd/4.0/

\section{LICENCE}

CC BY-NC-ND 4.0

\section{REPOSITORY RECORD}

Hopkins, Paul, Andrew J. Archer, and Robert Evans. 2019. "Pair-correlation Functions and Phase Separation in a Two-component Point Yukawa Fluid”. figshare. https://hdl.handle.net/2134/16036. 


\section{A 1 The Journal of Chemical Physics}

Pair-correlation functions and phase separation in a two-component point Yukawa fluid

P. Hopkins, A. J. Archer, and R. Evans

Citation: The Journal of Chemical Physics 124, 054503 (2006); doi: 10.1063/1.2162884

View online: http://dx.doi.org/10.1063/1.2162884

View Table of Contents: http://scitation.aip.org/content/aip/journal/jcp/124/5?ver=pdfcov

Published by the AIP Publishing

\section{Articles you may be interested in}

Interfacial and wetting properties of a binary point Yukawa fluid

J. Chem. Phys. 129, 214709 (2008); 10.1063/1.3027515

Microphase separation in multigraft copolymer melts studied by random-phase approximation and self-consistent field theory

J. Chem. Phys. 129, 114905 (2008); 10.1063/1.2980052

Phase separation of a binary two-dimensional core-softened fluid

J. Chem. Phys. 124, 234506 (2006); 10.1063/1.2202852

Dynamics of phase separation in confined twocomponent fluid membranes

AIP Conf. Proc. 708, 136 (2004); 10.1063/1.1764091

Solvent-mediated interactions and solvation close to fluid-fluid phase separation:A density functional treatment J. Chem. Phys. 118, 9726 (2003); 10.1063/1.1570406

\section{AIP $\mid$ chaos CALL FOR APPLICANTS Seeking new Editor-in-Chief}




\title{
Pair-correlation functions and phase separation in a two-component point Yukawa fluid
}

\author{
P. Hopkins, ${ }^{\text {a) }}$ A. J. Archer, ${ }^{\text {b) }}$ and R. Evans \\ H. H. Wills Physics Laboratory, University of Bristol, Bristol BS8 1TL, United Kingdom
}

(Received 19 September 2005; accepted 6 December 2005; published online 2 February 2006)

\begin{abstract}
We investigate the structure of a binary mixture of particles interacting via purely repulsive point Yukawa pair potentials with a common inverse screening length $\lambda$. Using the hypernetted chain closure to the Ornstein-Zernike equations, we find that for a system with "ideal" (Berthelot mixing rule) pair-potential parameters for the interaction between unlike species, the asymptotic decay of the total correlation functions crosses over from monotonic to damped oscillatory on increasing the fluid total density at fixed composition. This gives rise to a Kirkwood line in the phase diagram. We also consider a "nonideal" system, in which the Berthelot mixing rule is multiplied by a factor $(1+\delta)$. For any $\delta>0$ the system exhibits fluid-fluid phase separation and remarkably the ultimate decay of the correlation functions is now monotonic for all (mixture) state points. Only in the limit of vanishing concentration of either species does one find oscillatory decay extending to $r=\infty$. In the nonideal case the simple random-phase approximation provides a good description of the phase separation and the accompanying Lifshitz line. (C) 2006 American Institute of Physics.
\end{abstract}

[DOI: $10.1063 / 1.2162884]$

\section{INTRODUCTION}

A large class of fluids can be described generically as "big charged particles immersed in a neutralizing medium of lighter particles." Examples include charged colloidal suspensions ${ }^{1}$ and dusty plasmas. $^{2}$ A simple model for such systems describes the effective interaction between the bigger particles in terms of a Yukawa (screened Coulomb) pair potential $\phi(r) \propto \exp (-\lambda r) / r$. The effects of the screening due to the neutralizing medium are incorporated via the screening parameter $\lambda$. Such a Yukawa potential arises in, for example, the linearized Poisson-Boltzmann or DerjaguinLandau-Verwey-Overbeek theories for the effective potential between spherical charged colloids in solution. ${ }^{1}$ The effect of the neutralizing medium on the effective potential involves more than the screening effect as described by the parameter $\lambda$. There is an additional effect of charge renormalization whereby the amplitude of the effective potential $\phi(r)$ is not as one might perhaps expect from a linear treatment proportional to $Z^{2}$, where $Z$ is the charge on the big particles (colloids) rather the amplitude of $\phi(r)$ is proportional to $\bar{Z}^{2}$, where $\bar{Z}<Z$ is the renormalized charge. ${ }^{1}$

In the present paper we are concerned with a simple model of a binary mixture of big charged particles, with both species carrying charges of the same sign, immersed in a neutralizing background medium. For a general binary mixture of point Yukawa particles the pair potentials are dependent on six species specific parameters. These are the dimensionless coupling parameters $M_{i j}$ and the screening parameters $\lambda_{i j}$ for $i, j=1,2$. We assume that both species experience the same screening, $\lambda_{i j}=\lambda$, determined by the

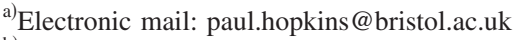

${ }^{b)}$ Electronic mail: andrew.archer@bristol.ac.uk
}

background medium (solvent), but that they have different coupling strengths. We can consider the coupling parameters to be proportional to the product of the effective charges of the species. Thus we define the pair potential as

$$
\phi_{i j}(r)=\frac{M_{i j} \epsilon \exp (-\lambda r)}{\lambda r},
$$

where $\epsilon$ denotes the overall energy scale, and all three potentials are repulsive: $M_{i j}>0$. As usual it is assumed that the interspecies parameters are related to those for like particles. ${ }^{3}$ The Berthelot mixing rule sets $M_{12}=\sqrt{M_{11} M_{22}}$. This choice could correspond to ions with charge $\bar{Z}_{i}$, with $\bar{Z}_{1}$ and $\bar{Z}_{2}>0$, immersed in a medium with an inverse screening length $\lambda$. In order to generalize this mixing rule we introduce a nonideality parameter $\delta>0$ such that $M_{12}=(1+\delta) \sqrt{M_{11} M_{22}}$. The case $\delta=0$ clearly corresponds to the ideal system. The nonideal case $\delta \neq 0$ can be viewed as arising from charge screening effects in the double layer of condensed counter ions on the surface of the particles. The double layer leads to an effective renormalization of the particle charge and strongly affects the particle interactions. ${ }^{1}$ One should expect that for some positive $\delta$ that the energy penalty incurred for unlike species to be neighbors should lead to fluid-fluid demixing at high densities; such behavior is found in models of soft-core fluids where positive nonadditivity gives rise to demixing. ${ }^{4-7}$

Binary mixtures of $\mathrm{H}^{+}$and $\mathrm{He}^{++}$in a neutralizing medium are expected to phase separate at temperatures and pressures of astrophysical interest-see Ref. 8. In this system the phase separation is thought to be due to charge neutralization being less efficient in the mixture than in the pure phases. We believe that some of the complex screening effects associated with such systems may be incorporated in a simple model such as ours, via the parameter $\delta$. Nonideal charge renormalization effects may also be present in binary 
suspensions of colloids. Charge renormalization may be affected by the local concentrations of the different species of colloids and counterions in very subtle ways leading to the possibility that the amplitude of $\phi_{12}(r)$ may not be simply proportional to $\bar{Z}_{1} \bar{Z}_{2}$. Such effects would be mimicked using our simple model. It may be the case that in some colloidal fluid mixtures $\delta>0$, or it may be that in other cases $\delta<0$, i.e., nonideal charge renormalization effects may favor mixing. However, in the present paper we investigate only the cases of $\delta=0$ and $\delta>0$. Using the accurate hypernetted chain (HNC) approximation we find that for the case $\delta=0$ the total pair-correlation functions $h_{i j}(r)$ exhibit crossover from monotonic to exponentially damped oscillatory asymptotic decay, $r \rightarrow \infty$, on increasing the total density of the fluid mixture at fixed composition. This scenario is equivalent to that observed in the one-component point Yukawa fluid (OCY). ${ }^{9}$ However, in the nonideal case, an infinitesimal positive $\delta$ can give rise to fluid-fluid phase separation at a sufficiently large total density. Moreover we find that the fluid structure is changed profoundly from that pertaining to $\delta=0$, i.e., for all thermodynamic state points, apart from the limits of pure species 1 and 2, the ultimate, $r \rightarrow \infty$, decay of correlations is monotonic. We find that for the particular choice $\delta=0.1$ the very simple random-phase approximation (RPA) provides a good account of the fluid-fluid binodal and spinodal obtained from the HNC but a poor account of the detailed behavior of the correlation functions.

The paper is arranged as follows: In Secs. II and III we remind readers of the HNC and RPA integral equations and some basic results from the theory of the asymptotic decay of pair-correlation functions in binary mixtures. Section IV describes results for $h_{i j}(r)$ and for the poles of the Fourier transforms $\hat{h}_{i j}(q)$ obtained from numerical solutions of the HNC closure approximation. Within the RPA we are able to calculate the corresponding poles analytically and we compare these results with those from the HNC. The pole analysis enables us to determine the behavior of $h_{i j}(r)$ at the intermediate range, as well as at the longest range, $r \rightarrow \infty$. Particular attention is paid to the behavior of the correlation functions in the limit where the density of species $2, \rho_{2} \rightarrow 0$. In Sec. $\mathrm{V}$ we present phase diagrams, in the composition, total density plane, along with Lifshitz lines for the partial structure factors, obtained from both the HNC and RPA. We draw some conclusions in Sec. VI.

\section{CLOSURE OF THE ORNSTEIN-ZERNIKE (OZ) EQUATIONS}

Our starting point for determining the fluid structure is the mixture Ornstein-Zernike $(\mathrm{OZ})$ equations ${ }^{3}$ which relate the total correlation functions $h_{i j}(r)=g_{i j}(r)-1$, where $g_{i j}(r)$ are the radial distribution functions, to a set of pair direct correlation functions $c_{i j}(r)$,

$$
h_{i j}\left(r_{12}\right)=c_{i j}\left(r_{12}\right)+\sum_{k=1}^{2} \rho_{k} \int d^{3} r_{3} c_{i k}\left(r_{13}\right) h_{k j}\left(r_{32}\right),
$$

where $r_{i j}=\left|\mathbf{r}_{i}-\mathbf{r}_{j}\right|$ and $\rho_{k}$ is the bulk density of species $k$. These equations can be viewed as defining the pair direct correlation functions. In order to determine the fluid structure a second relation, or closure, is required.

The simplest closure germane to the present model is the RPA: $c_{i j}^{\mathrm{RPA}}=-\beta \phi_{i j}(r)$ with $\beta=\left(k_{B} T\right)^{-1}$, which is strictly valid only for $r \rightarrow \infty$. Although this approximation is inadequate for hard-core model systems, it has been shown that the RPA becomes accurate for some soft-core systems at intermediate densities and exact at high densities. ${ }^{4-7,10-12}$ An important advantage of the RPA is that it does provide an analytical solution for correlation functions and for thermodynamic properties which may provide valuable physical insight into fluid behavior.

A more accurate approximation is the HNC approximation. The exact closure of the $\mathrm{OZ}$ equations can be expressed as

$$
g_{i j}(r)=\exp \left(-\beta \phi_{i j}(r)+h_{i j}(r)-c_{i j}(r)-b_{i j}(r)\right),
$$

where $-b_{i j}(r)$ is an unknown bridge function. ${ }^{3}$ The HNC simply sets this bridge function to zero for all $r$. It is found to be accurate for long-ranged or soft-core potentials ${ }^{3,8,10}$ although it may fail in the neighborhood of a spinodal. For the one-component (point) Yukawa fluid it is known that the HNC is remarkably accurate for small coupling parameters. ${ }^{9,13}$ In order to determine the correlation functions within the $\mathrm{HNC}$ we use a standard iterative procedure. In what follows, we fix the reduced temperature $T^{*}=(\beta \epsilon)^{-1}$ so that the state of the system is determined by the total density $\rho=\rho_{1}+\rho_{2}$, and the species concentrations $x_{i}=\rho_{i} / \rho$.

\section{ASYMPTOTIC DECAY OF CORRELATION FUNCTIONS}

There are two procedures for determining the asymptotic, $r \rightarrow \infty$, behavior of the total correlation functions. One is to examine directly the numerical solutions for $h_{i j}(r)$. The alternative method is to input the direct correlation functions (in the present case from either the RPA or HNC closures) into the set of $\mathrm{OZ}$ equations (2) and perform an asymptotic analysis. The $\mathrm{OZ}$ equations can be solved formally in Fourier space and the solution written as

$$
\hat{h}_{i j}(q)=\frac{N_{i j}(q)}{D(q)}
$$

where $\hat{h}_{i j}(q)$ denotes the three-dimensional Fourier transform of $h_{i j}(r)$. The three functions share the same denominator,

$$
D(q)=\left[1-\rho_{1} \hat{c}_{11}(q)\right]\left[1-\rho_{2} \hat{c}_{22}(q)\right]-\rho_{1} \rho_{2} \hat{c}_{12}(q)^{2},
$$

but the numerators depend on the particular indices:

$$
\begin{aligned}
& N_{11}(q)=\hat{c}_{11}(q)+\rho_{2}\left[\hat{c}_{12}(q)^{2}-\hat{c}_{11}(q) \hat{c}_{22}(q)\right], \\
& N_{22}(q)=\hat{c}_{22}(q)+\rho_{1}\left[\hat{c}_{12}(q)^{2}-\hat{c}_{11}(q) \hat{c}_{22}(q)\right], \\
& N_{12}(q)=N_{21}(q)=\hat{c}_{12}(q) .
\end{aligned}
$$




$$
r h_{i j}(r)=\frac{1}{2 \pi^{2}} \int_{0}^{\infty} d q q \sin (q r) \hat{h}_{i j}(q)
$$

Using Eq. (4) and assuming that the singularities of $\hat{h}_{i j}(q)$ for the present Yukawa systems are simple poles we are able to proceed via the residue theorem. ${ }^{14}$ Performing contour integration around a semicircle in the upper half of the complex $q$ plane, we write the total correlation functions as a sum of contributions from the poles enclosed,

$$
r h_{i j}(r)=\sum_{n} A_{n}^{i j} \exp \left(\mathrm{i} q_{n} r\right),
$$

where $q_{n}$ satisfies $D\left(q_{n}\right)=0$ and $A_{n}^{i j}$ is the amplitude associated with the pole $q_{n}$. This amplitude is related to the residue $R_{n}^{i j}$ of $q N_{i j}(q) / D(q)$ by $A_{n}^{i j}=R_{n}^{i j} / 2 \pi$. The poles are either purely imaginary, $q=i \alpha_{0}$, or occur as a conjugate pair $q$ $= \pm \alpha_{1}+\mathrm{i} \widetilde{\alpha}_{0}{ }^{14}$

In general there is an infinite number of poles and contributions from many of these are required to account for the behavior of $h_{i j}(r)$ at small $r$. However, the ultimate, $r \rightarrow \infty$, decay of $h_{i j}(r)$ is determined by the pole that gives the slowest exponential decay, i.e., the pole with the smallest imaginary part. This is referred to as the leading order pole. If the leading order pole is purely imaginary then $r h_{i j}(r)$ decays exponentially, $r h_{i j}(r) \sim A_{i j} \exp \left(-\alpha_{0} r\right)$, as $r \rightarrow \infty$. On the other hand, if the leading order poles are a conjugate pair, then the sum of contributions from this pair of complex poles gives damped oscillatory decay, $r h_{i j}(r) \sim 2 \widetilde{A}_{i j} \exp \left(-\widetilde{\alpha}_{0} r\right) \cos \left(\alpha_{1} r-\widetilde{\theta}_{i j}\right)$, where $\widetilde{A}_{i j}$ and $\widetilde{\theta}_{i j}$ denote the amplitude and phase, respectively. ${ }^{14}$

Note that whereas the wavelength $2 \pi / \alpha_{1}$ and the decay lengths $\alpha_{0}^{-1}$ or $\widetilde{\alpha}_{0}^{-1}$ are the same for all $h_{i j}(r)$, the amplitudes and phases do depend on the indices $i j .{ }^{14}$ However, general considerations demand $A_{12}^{2}=A_{11} A_{22}$ or $\widetilde{A}_{12}^{2}=\widetilde{A}_{11} \widetilde{A}_{22}$ and $2 \widetilde{\theta}_{12}$ $=\tilde{\theta}_{11}+\tilde{\theta}_{22} \cdot{ }^{14}$ In the next section we shall employ the RPA and the HNC closures to investigate the ultimate, $r \rightarrow \infty$, decay of $h_{i j}(r)$ and the behavior of $h_{i j}(r)$ at intermediate distances $r$. The values of the amplitudes are relevant in determining which pole or complex-conjugate pair provides the dominant contribution to $h_{i j}(r)$ in the intermediate regime.

\section{RESULTS FOR PAIR-CORRELATION FUNCTIONS}

In Fig. 1 we display the radial distribution functions $g_{i j}(r)$, obtained from the HNC closure, for the state point $\rho \lambda^{-3}=0.5, x_{2}=0.5$, and $T^{*}=1$. The coupling parameters are fixed at $M_{11}=1$ and $M_{22}=4$. Figure 1(a) corresponds to ideal (Berthelot) mixing $\delta=0$; Fig. 1(b) has $\delta=10^{-5}$, weak nonideality, while Fig. 1(c) has $\delta=0.1$. In all cases the $g_{i j}(r)$ appear structureless but the expanded scales of the insets reveal the nature of the intermediate and long-range decay of the paircorrelation functions. For $\delta=0, r h_{i j}(r)$ shows an exponentially damped oscillatory decay extending to arbitrarily large separations $r$. By contrast, for $\delta=10^{-5}$, there are several oscillations at small $r$ and $r h_{i j}(r)$ decays monotonically (exponentially) for $\lambda r \gtrsim 8$. For $\delta=0.1$ the monotonic decay extends from $\lambda r \simeq 3$. Clearly the choice of $\delta$ has a profound influence on the behavior of the pair-correlation functions. If one fixes the concentration at $x_{2}=0.5$ and reduces the total density $\rho$
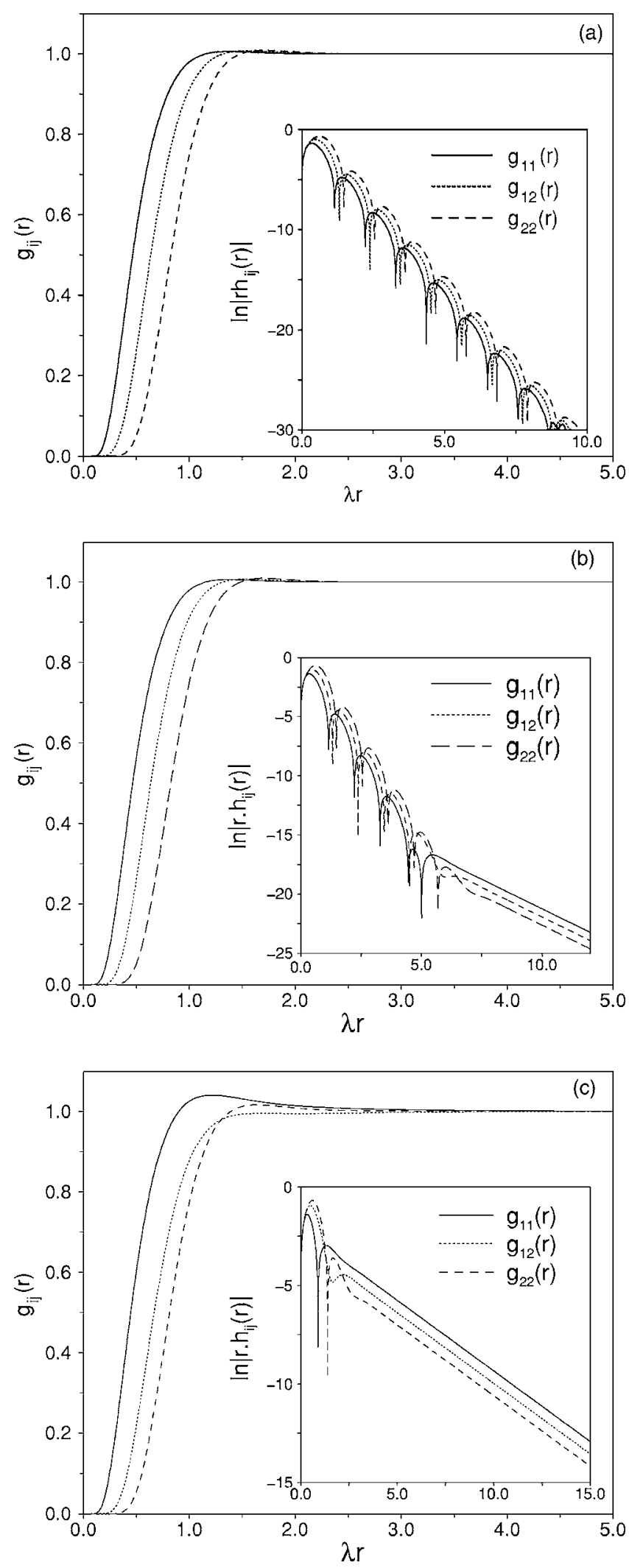

FIG. 1. Pair-correlation functions for the state point $\rho \lambda^{-3}=0.5, x_{2}=0.5$, and $T^{*}=1$, calculated from the HNC closure, using the coupling parameter ratio $M_{22} / M_{11}=4$. Main figures show radial distribution functions $g_{i j}(r)$ and insets show $\ln \left|r h_{i j}(r)\right|$ vs $\lambda r$. (a) $\delta=0$. Exponentially damped oscillations extend to infinity. (b) $\delta=10^{-5}$. For $\lambda r \gtrsim 8, r h_{i j}(r)$ exhibit monotonic (exponential) decay. (c) $\delta=0.1$. Monotonic decay now develops for $\lambda r \gtrsim 3$. 
one finds that for $\delta=0$ there is a crossover from the exponentially damped oscillatory behavior of $r h_{i j}(r)$ shown in Fig. 1(a) to monotonic (exponential) decay similar to that shown in Fig. 1(c), near $\rho \lambda^{-3} \simeq 0.05$. Such crossover is found in the OCY on reducing the density at fixed $T^{*}{ }^{9}$ The scenario is different for $\delta>0$. On reducing the density at fixed $x_{2}=0.5$ the $h_{i j}(r)$ are similar to those for $\rho \lambda^{-3}=0.5$; the decay remains monotonic. Similar features are found for other values of the concentration provided $x_{1}$ and $x_{2} \neq 0$. If $x_{2}=0$ or 1 then one recovers the OCY which exhibits crossover as mentioned above.

In order to understand these results emanating from the full numerical solution of the HNC closure we turn to the asymptotic (pole) analysis of Sec. III. It is convenient to begin with the simple RPA treatment before discussing the more sophisticated $\mathrm{HNC}$ results.

\section{A. Poles in the RPA}

The advantage of the RPA is that the pair direct correlation functions and their Fourier transforms $\hat{c}_{i j}(q)$ are given analytically. This means that the poles can be determined analytically. Using the definition of the RPA it follows from Eq. (1) that

$$
\hat{c}_{i j}^{\mathrm{RPA}}(q)=-\frac{4 \pi M_{i j}}{\lambda T^{*}} \frac{1}{\left(\lambda^{2}+q^{2}\right)} .
$$

Substituting this form into Eq. (5) we can solve for the zeros of $D(q)$, i.e, the poles $q_{n}$ at each state point. We find that within the RPA there are only two poles. Both are purely imaginary and are given by

$$
\alpha_{0}=\sqrt{\frac{2 \pi \rho}{\lambda T^{*}}\left(M_{0} \pm \sqrt{M_{0}^{2}+M_{\delta}}\right)+\lambda^{2}},
$$

where we have introduced $M_{0}=x_{1} M_{11}+x_{2} M_{22}$ and $M_{\delta}$ $=4 x_{1} x_{2} M_{11} M_{22}(2+\delta) \delta$.

For $\delta=0$ this gives an imaginary pole with $\alpha_{0}^{+} \geqslant \lambda$, pertaining to the positive sign, and a "false" solution obtained with the negative sign giving $\alpha_{0}=\lambda$ for all state points. The imaginary pole is analogous to that found in the RPA solution for the OCY. Figure 2 shows how this pole ascends from $\alpha_{0}=\lambda$ at $\rho=0$ as the density $\rho$ is increased, along a line of constant concentration, $x_{2}=0.5$, at a fixed temperature $T^{*}=1$.

By calculating the residues one can show [see Eq. (14) below] that the false pole $\alpha_{0}=\lambda$ makes no contribution to $h_{i j}(r)$, i.e, for $\delta=0$ the corresponding amplitude $A_{i j}^{-}=0$ and

$$
r h_{i j}^{\mathrm{RPA}}(r)=A_{i j}^{+} \exp \left(-\alpha_{0}^{+} r\right),
$$

where $\alpha_{0}^{+}$is given by Eq. (10), with the positive sign, and the amplitudes are independent of the density, ${ }^{15}$

$$
A_{i j}^{+}=-\frac{M_{i j}}{\lambda T^{*}}
$$

The situation is quite different for $\delta>0$. We find that the pole with $\alpha_{0}^{+}>\lambda$ is modified slightly by the addition of a (relatively) small positive term. More significantly we find that the solution with the negative sign now corresponds to a second pole which descends from $\alpha_{0}^{-}=\lambda$ eventually reaching

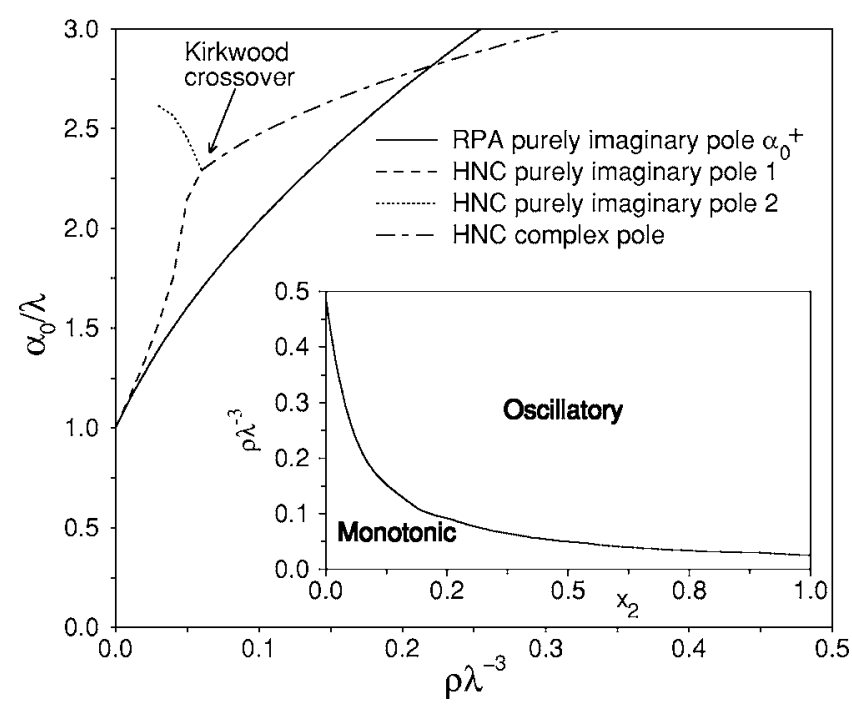

FIG. 2. The imaginary part of leading order poles $\alpha_{0}$ calculated along a path in the phase diagram of increasing density $\rho$ for fixed $x_{2}=0.5$ and $T^{*}=1 . \delta$ $=0$, corresponding to an "ideal" mixture. The RPA solution, Eq. (10), consists of a single, purely imaginary pole $\alpha_{0}^{+}$which increases steadily with density from $\alpha_{0} / \lambda=1$. Within the HNC closure, at low densities $\rho$, the two leading order poles are both purely imaginary. As the density is increased the first imaginary pole ascends and the second imaginary pole descends and, at the Kirkwood crossover point, coalesce becoming a conjugate pair $q= \pm \alpha_{1}+i \alpha_{0}$. Increasing the density further causes both the real and imaginary parts of the complex poles to increase. Thus the Kirkwood point marks the boundary between monotonic (at small $\rho \lambda^{-3}$ ) and damped oscillatory asymptotic decay. The inset shows the Kirkwood line, separating the two types of decay, plotted in the concentration-total-density phase diagram.

zero as the density is increased-see Fig. 3. The locus of points in the phase diagram for which $\alpha_{0}^{-}=0$ is the spinodal, where the $\hat{h}_{i j}(q)$ (or the partial structure factors) diverge in the limit $q \rightarrow 0$, indicating that the system is undergoing fluid-fluid demixing. Note that this second "spinodal" pole exists only for the mixture states, i.e., for $x_{1}$ and $x_{2} \neq 0$; in the pure states it reverts to the false solution $\alpha_{0}=\lambda$. The behavior of this pole is shown for fixed $x_{2}=0.5$, and increasing density in Fig. 3.

The spinodal pole is present for arbitrarily small positive $\delta$. Therefore the RPA predicts that the system will undergo phase separation provided that there is any degree of positive nonideality in the pair potentials. This behavior is reflected in the asymptotic decay of the total correlation functions. For $\delta>0$,

$$
r h_{i j}^{\mathrm{RPA}}(r)=A_{i j}^{+} \exp \left(-\alpha_{0}^{+} r\right)+A_{i j}^{-} \exp \left(-\alpha_{0}^{-} r\right),
$$

with $\alpha_{0}^{-}<\lambda<\alpha_{0}^{+}$, which follows from Eq. (10). The particularly simple forms of Eqs. (11) and (13) are a consequence of the RPA. ${ }^{15}$ The amplitudes are

$$
A_{i j}^{ \pm}=-\frac{1}{4 \lambda T^{*}} \frac{2 M_{i j}\left(M_{0} \pm \sqrt{M_{0}^{2}+M_{\delta}}\right)+\delta_{i j} M_{\delta} x_{i}}{M_{0}+M_{\delta}\left(M_{0} \pm \sqrt{M_{0}^{2}+M_{\delta}}\right)},
$$

where superscript \pm refers to the sign in front of the square root and we use the compound parameters $M_{0}$ and $M_{\delta}$ defined previously. $\delta_{i j}$ is the Kronecker delta. As the ultimate decay is determined by the pole with the smallest imaginary part, the spinodal pole $\alpha_{0}^{-}$must determine the ultimate decay of $h_{i j}(r)$ for any $\delta>0$. For very small $\delta>0, \alpha_{0}^{-}$lies very 


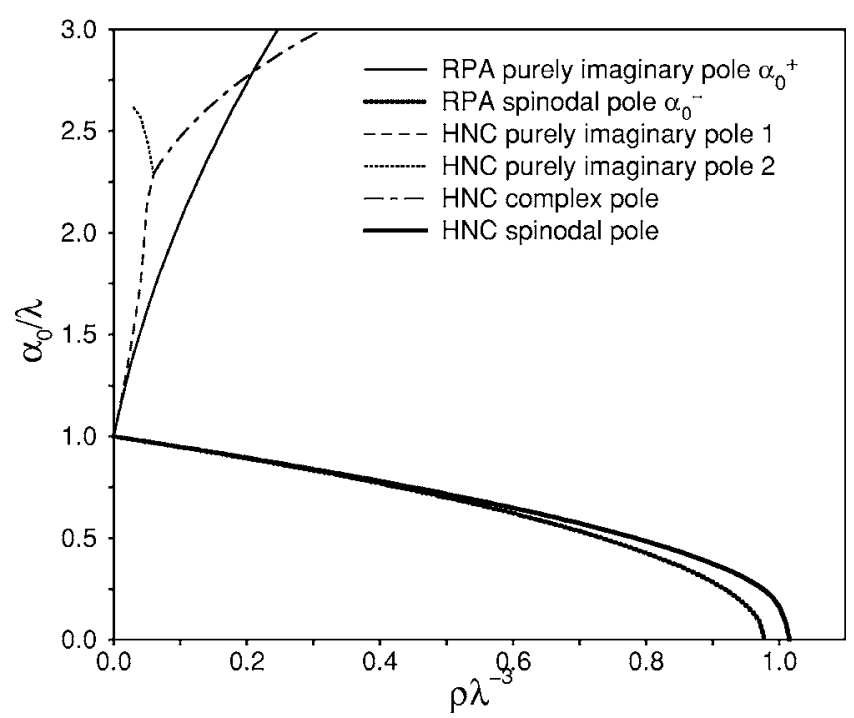

FIG. 3. As in Fig. 2 but now for $\delta=0.1$. Within the RPA the imaginary pole $\alpha_{0}^{+}$is shifted by only a small amount from the case $\delta=0$. A second purely imaginary pole $\alpha_{0}^{-}$is introduced which decreases from $\lambda$ to zero as $\rho$ is increased; $\alpha_{0}^{-}=0$ corresponds to the spinodal. Since $\alpha_{0}^{-}<\alpha_{0}^{+}$this "spinodal" pole determines the ultimate decay of the total correlation functions. Within the HNC closure the Kirkwood mechanism is still present and the crossover point is largely unchanged from the case $\delta=0$. However, a spinodal pole is introduced which follows closely the corresponding RPA pole $\alpha_{0}^{-}$. It follows that for all densities the ultimate decay of the total correlation functions is monotonic. Although the Kirkwood crossover is present it does not involve the leading order spinodal pole and therefore does not influence the ultimate decay.

close to $\lambda$ until the reduced density $\rho \lambda^{-3}$ reaches very large values, i.e., the spinodal is shifted to very large densities as $\delta \rightarrow 0$. In these circumstances the relative magnitude of the amplitudes $A_{i j}^{+}$and $A_{i j}^{-}$becomes important; note that $\alpha_{0}^{+}$depends only weakly on $\delta$. For a given state point the amplitude of the spinodal pole $A_{i j}^{-}$decreases relative to $A_{i j}^{+}$as $\delta \rightarrow 0$ and one must go to increasing values of $r$ before the spinodal pole provides the dominant contribution to $h_{i j}(r)$. At intermediate $r$ the contribution from $\alpha_{0}^{+}$determines the decay behavior.

\section{B. Poles in the HNC}

For the OCY the HNC predicts a crossover line in the $(\rho, T)$ plane separating a region where the asymptotic decay of $h(r)$ is damped oscillatory from that where it is monotonic. The crossover takes place via the coalescence of two imaginary poles to form a complex pole as the density is increased at fixed temperature $T .^{9}$ The mechanism is equivalent to that discussed first by Kirkwood in pioneering studies of strong electrolytes. ${ }^{16}$ Thus for the pure species we take the results of Ref. 9 and read off the crossover values of $\rho \lambda^{-3}$. For $T^{*}=1$ and $x_{2}=0$ (pure species 1) crossover occurs at $\rho \lambda^{-3} \approx 0.47$ whereas for $x_{2}=1$ (pure species 2) this occurs at $\rho \lambda^{-3} \approx 0.025$. If we vary the concentration between these limiting values (at fixed $T^{*}=1$ ) we expect to find a crossover line in the $\left(x_{2}, \rho \lambda^{-3}\right)$ plane. We determined this line by calculating the poles of $\hat{h}_{i j}(q)$, i.e., the zeros of $D(q)$, using the same numerical procedure as in Ref. 9 for the OCY.

The pair direct correlation functions $c_{i j}(r)$ are obtained from the full solutions of the HNC integral equations. In order to ensure convergence of the integrals which determine the poles we follow the procedure given in Refs. 9 and 17. For $r \rightarrow \infty, c_{i j}(r) \rightarrow-M_{i j} \exp (-\lambda r) /\left(\lambda T^{*} r\right)$, so we define a short-range direct correlation function $c_{i j}^{\mathrm{sr}}(r)$,

$$
c_{i j}^{\mathrm{sr}}(r) \equiv c_{i j}(r)+\frac{M_{i j} \exp (-\lambda r)}{\lambda T^{*} r} .
$$

Fourier transforming we find

$$
\hat{c}_{i j}(q)=\hat{c}_{i j}^{\mathrm{sr}}(q)-\frac{4 \pi M_{i j}}{\lambda T^{*}} \frac{1}{\left(q^{2}+\lambda^{2}\right)},
$$

which can be substituted into Eq. (5). $D(q)$ is separated into its real and imaginary components, and the equation $D(q)=0$ is solved numerically using a Newton-Raphson procedure. In general, the relevant integrals converge only for complex $q$ such that $\Im[q] \leqslant 2 \alpha_{0}$, where $\alpha_{0}$ is the imaginary part of the leading order pole. ${ }^{17}$ It follows that only a few poles can be calculated; the remaining poles are situated outside this region of convergence. Fortunately the poles relevant for determining the basic features of the decay of correlations can be obtained.

For the case $\delta=0$ we find that for all $x_{2}$ the leading poles exhibit the Kirkwood crossover mimicking that in the OCY. ${ }^{9}$ At very low densities we find an imaginary pole (dashed line in Fig. 2) that ascends from $\alpha_{0}=\lambda$ as we increase the density $\rho$ at constant concentration, and is initially similar to the RPA. At slightly higher densities a second imaginary pole (dotted line) moves into the region of convergence and descends as $\rho$ increases. These two poles move towards each other and then, at the Kirkwood point, coalesce. On increasing the density further, one finds a conjugate pair whose real and imaginary parts both increase with $\rho$ (dash-dotted line). Figure 2 shows the imaginary parts of the relevant poles as they undergo the Kirkwood crossover. The locus of points for which the poles coalesce, the Kirkwood line, is shown in the inset to Fig. 2. Below this line the asymptotic decay of $r h_{i j}(r)$ is pure exponential and above the line it is exponentially damped oscillatory. One sees that the state point $x_{2}$ $=0.5$ and $\rho \lambda^{-3}=0.5$, which corresponds to the results for $h_{i j}(r)$ in Fig. 1(a), lies deep within the oscillatory region so one expects to find oscillations extending to $r \rightarrow \infty$.

Figure 3 displays the corresponding plots of $\alpha_{0}$ for $\delta=0.1$. Now there are three purely imaginary poles at low densities. Two of these mimic closely what is found for $\delta=0$, i.e, they coalesce and form a complex pole at a Kirkwood point that is not far removed from the corresponding point for $\delta=0$. Once again one of these poles (dashed line) lies close to the RPA solution $\alpha_{0}^{+}$for very small values of $\rho \lambda^{-3}$. The third pole follows closely the RPA spinodal pole $\alpha_{0}^{-}$; it decreases slowly with increasing $\rho \lambda^{-3}$ for small densities before decreasing rapidly to zero at the spinodal point which is at $\rho \lambda^{-3} \approx 1.01$, slightly higher than the value $\rho \lambda^{-3}$ $=0.98$ found from the RPA. We refer to the third pole as the HNC spinodal pole.

It is evident from Fig. 3 that within the $\mathrm{HNC}$, as in the RPA, the purely imaginary spinodal pole with $\alpha_{0}<\lambda$ will determine the ultimate decay of $h_{i j}(r)$ which will be mono- 


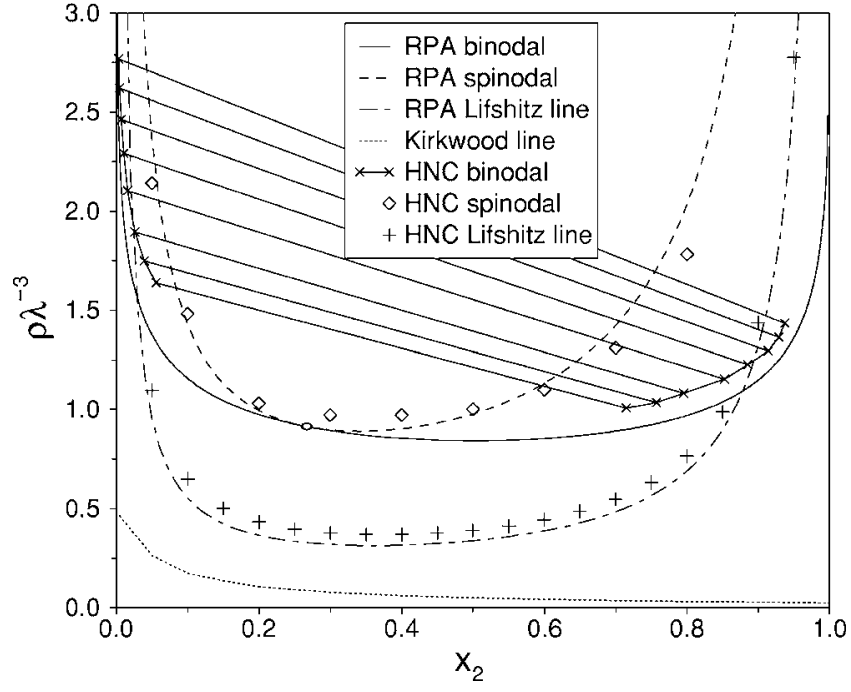

FIG. 4. Phase diagram for the binary Yukawa system with $M_{22} / M_{11}$ $=4, T^{*}=1$, and $\delta=0.1$. The system exhibits phase separation within both the RPA and HNC closures. The RPA spinodal (dashed curve) meets the RPA binodal (solid curve) at the critical point $O$. The HNC spinodal points (diamonds) were calculated by extrapolating the spinodal pole to zero along lines of constant concentration and increasing density. The HNC binodal was calculated using the method outlined in the text; the straight segments are tie lines connecting coexisting fluid phases denoted by crosses and correspond to the following reduced pressures: $\beta P \lambda^{-3}=20,22,25,30,35,40$, 45 , and 50. Also shown is the Lifshitz line for $S_{\mathrm{NN}}(q)$ calculated in both the RPA (dash dotted) and HNC (crosses). The dotted line at low density denotes the Kirkwood crossover from monotonic to oscillatory decay of correlation functions. However, for $\delta>0$ and $0<x_{2}<1$ the true asymptotic decay remains monotonic even for states above the line-see text.

tonic for all densities provided that $0<x_{2}<1$. The ultimate decay cannot be oscillatory. Although there is still a Kirkwood-type crossover, this now involves higher-order poles rather than the leading order poles, which was the case for $\delta=0$. The corresponding crossover line is shown as the dotted line in the $\left(x_{2}, \rho \lambda^{-3}\right)$ phase diagram for $\delta=0.1$ displayed in Fig. 4. Note that the line lies far below the HNC spinodal (diamonds). Crossover does not influence the ultimate decay of $h_{i j}(r)$ which is stubbornly monotonic.

Of course, this does not mean that we do not find oscillations at intermediate $r$. For densities larger than the crossover value the HNC pole analysis predicts, for $r \rightarrow \infty$,

$$
r h_{i j}(r) \sim A_{i j} \exp \left(-\alpha_{0} r\right)+2 \widetilde{A}_{i j} \exp \left(-\widetilde{\alpha}_{0} r\right) \cos \left(\alpha_{1} r-\widetilde{\theta}_{i j}\right)
$$

with $\alpha_{0}<\widetilde{\alpha}_{0}$. Provided $\widetilde{A}_{i j} \gg A_{i j}$ and we are not close to the spinodal $\left(\alpha_{0}=0\right)$ the oscillatory contribution will be significant in the intermediate regime.

An example is given in Fig. 5 which compares the full numerical result for $h_{22}(r)$ with the contribution given by Eq. (17) for a state point $\left(x_{2}=0.99, \rho \lambda^{-3}=0.05\right)$ where the amplitude of the spinodal pole is very small. On a normal scale plot $h_{22}(r)$ is well described, for $\lambda r \gtrsim 1.5$, by the contribution from a single conjugate pair of poles, i.e., by the second term in Eq. (17). This contribution is oscillatory, albeit with a small amplitude. The inset to Fig. 5 plots $\ln \left|r h_{22}(r)\right|$ for a larger range of $\lambda r$. Now the oscillations are apparent but we observe monotonic (exponential) decay, described accurately

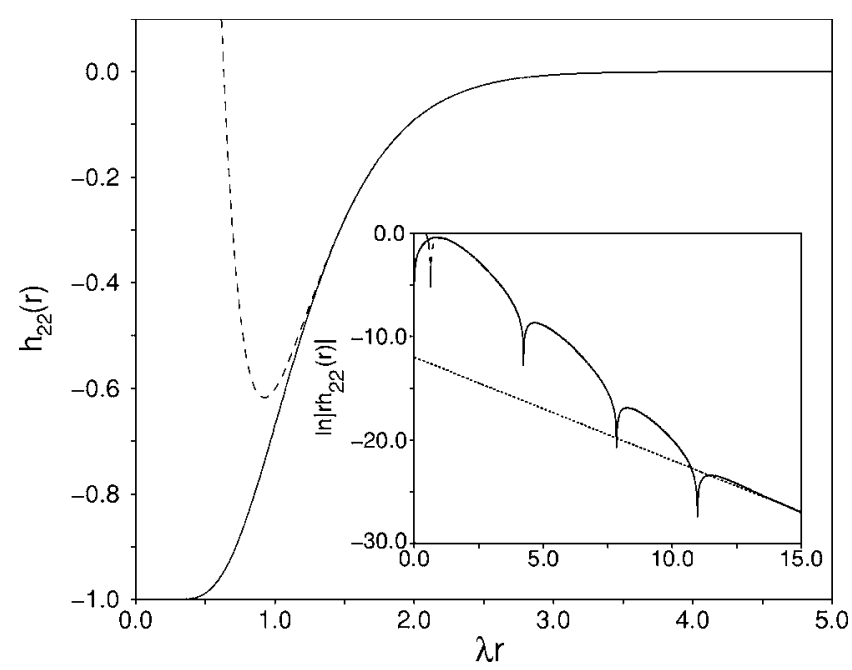

FIG. 5. Comparison between results of the full HNC solution for $h_{22}(r)$ (solid line) and the contributions of the lowest order poles for state point $\rho \lambda^{-3}=0.05, x_{2}=0.99$, and parameters $M_{22} / M_{11}=4, T^{*}=1$, and $\delta=0.1$. Note this point lies above the Kirkwood crossover line in Fig. 4. The main figure shows that the decay of $h_{22}(r)$ is well described by the contribution from a single pair of conjugate poles (dashed line) $\left(\widetilde{\alpha}_{0} / \lambda_{0}=2.269\right.$ and $\alpha_{1} / \lambda$ $=0.8723$ ). On this scale the contribution from the purely imaginary pole is vanishingly small. In the inset the plot of $\ln \left|r h_{22}(r)\right|$ vs $\lambda r$ (solid line) shows that although the oscillatory contribution (dashed line-visible only in top left hand corner) describes $h_{22}(r)$ accurately for intermediate range, the contribution from the "spinodal" pole (dotted line) $\left(\alpha_{0} / \lambda=0.9993\right)$ determines the decay for $\lambda r \gtrsim 14$. Thus the sum of contributions from the oscillatory and spinodal poles (dot dashed) given by Eq. (17) is very accurate in the range of $1.5<\lambda r<\infty$.

by the first term in Eq. (17), for $\lambda r \gtrsim 14$ (dotted line). As the amplitude $\lambda A_{22}$ of the spinodal pole contribution is $\sim 10^{-6}$ this contribution does not become significant until large separations $r$. Of course such a contribution might be impossible to detect experimentally or in simulations. We conclude that although a pair-correlation function might exhibit many oscillations, the ultimate decay can still be monotonic. Note that for a state point closer to the spinodal, such as in Fig. 1 (c) where $x_{2}=0.5, \rho \lambda^{-3}=0.5$, and $\alpha_{0} / \lambda \approx 0.72$, the purely exponential contribution in Eq. (17) dominates for $\lambda r \geqslant 3$.

For a pure (one component) fluid there is no spinodal pole; within the HNC the Kirkwood crossover occurs between leading order poles. Therefore, on the axes $x_{2}=0$ and $x_{1}=0$ in Fig. $4 h_{i i}(r)$ must decay in an oscillatory fashion as $r \rightarrow \infty$, provided we consider states above the Kirkwood crossover densities. However, as we increase $x_{2}$ (or $x_{1}$ ) infinitesimally the spinodal pole makes a nonvanishing contribution to the correlation functions and forces the ultimate decay of $h_{i j}(r)$ to be monotonic. In order to understand the evolution of this behavior we must consider the amplitudes $A_{i j}$ of the spinodal pole as the concentration $x_{2}$ vanishes along a line of constant total density. Figure 6 shows results for $\rho \lambda^{-3}=0.5$, a density that lies just above the Kirkwood crossover value, $\rho \lambda^{-3} \sim 0.47$, for the pure fluid. The spinodal pole $\alpha_{0} / \lambda \rightarrow 1^{-}$as $x_{2} \rightarrow 0$ and the accompanying amplitudes $A_{11}$ (positive) and $A_{12}$ (negative) both vanish, as power laws, in this limit. It follows that the spinodal pole makes contributions to $h_{11}(r)$ and $h_{12}(r)$ that become vanishingly small in a continuous fashion, as $x_{2} \rightarrow 0$. Thus for very small concen- 


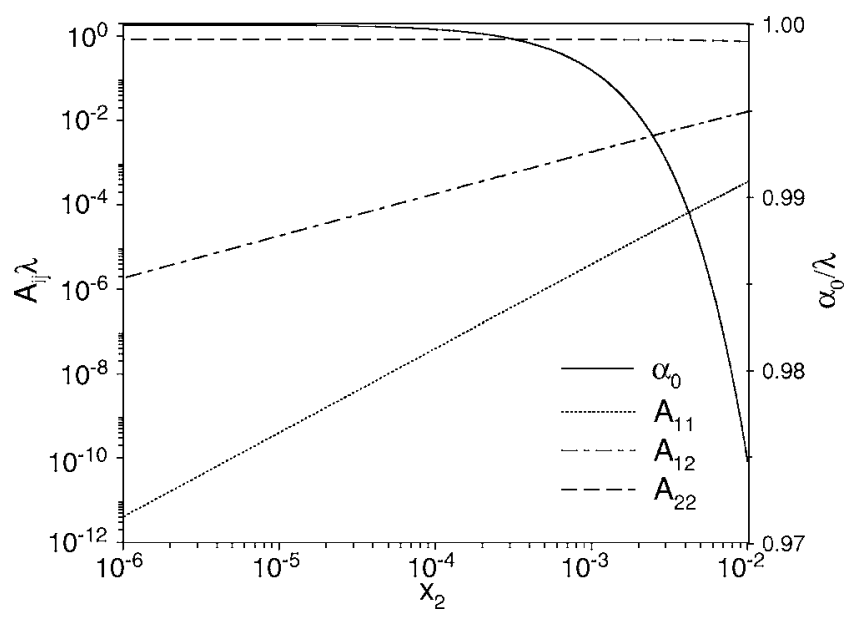

FIG. 6. The HNC "spinodal" pole $\alpha_{0}$ and corresponding amplitudes $A_{i j}$ as a function of concentration $x_{2}$ along a path of constant density $\rho \lambda^{-3}=0.5$, for $T^{*}=1$ and $\delta=0.1$. As $x_{2} \rightarrow 0, \alpha_{0} / \lambda \rightarrow 1^{-}$and $A_{11}, A_{12} \rightarrow 0$ but $A_{22} \lambda$ tends to 0.84 . Note that $A_{12}$ is negative and its magnitude is plotted here. The amplitudes obey the rule $A_{12}^{2}=A_{11} A_{22}$, see text.

trations $h_{11}(r)$ and $h_{12}(r)$ appear oscillatory until extremely large values of $\lambda r$ where the spinodal pole will dictate the final (monotonic) decay of these functions.

The amplitude $A_{22}$ of the minority component, species 2 , has a different variation. As shown in Fig. 6, $\lambda A_{22}$ remains almost constant as the concentration is reduced and tends to a nonzero value (0.84) at $x_{2}=0$. At first sight this result is a little surprising. However, we should recall that physical observables such as probability distributions or the liquid structure factors involve the product $\rho_{2} h_{22}(r)$ which vanishes as $x_{2} \rightarrow 0$. We confirmed that our numerical results for the amplitudes satisfy the rule ${ }^{14} A_{12}^{2}=A_{11} A_{22}$ mentioned in Sec. III.

At this point it is instructive to return to the RPA spinodal pole. Recall that $\alpha_{0}^{-}$is given by Eq. (10) and the corresponding amplitudes by Eq. (14). If we Taylor expand around $x_{2}=0$ we find that

$$
\alpha_{0}^{-}=\lambda\left(1-\frac{2 \pi \rho M_{22} \delta(2+\delta)}{\lambda^{3} T^{*}} x_{2}\right)+O\left(x_{2}^{2}\right),
$$

$A_{11}^{-}$and $A_{12}^{-}$decay to zero as power laws in $x_{2}$, and that $A_{22}^{-}$ tends to a constant, i.e.,

$$
\begin{aligned}
& A_{11}^{-}=\frac{M_{22}^{2}(1+\delta)^{2}(2+\delta) \delta}{\lambda T^{*} M_{11}} x_{2}^{2}+O\left(x_{2}^{3}\right), \\
& A_{12}^{-}=-\frac{M_{22}^{3 / 2}(1+\delta)(2+\delta) \delta}{\lambda T^{*} M_{11}^{1 / 2}} x_{2}+O\left(x_{2}^{2}\right), \\
& A_{22}^{-}=\frac{M_{22}(2+\delta) \delta}{\lambda T^{*}}+O\left(x_{2}\right) .
\end{aligned}
$$

In the limit $x_{2} \rightarrow 0, \lambda A_{22}^{-}=M_{22}(2+\delta) \delta / T^{*}$ which takes the value 0.84 for the present choice of parameters: $M_{22}=4$, $\delta=0.1$, and $T^{*}=1$. This is the same limiting value of the amplitude as obtained in the HNC. Indeed we find that the RPA results for $\alpha_{0}^{-}$and for the amplitudes $A_{i j}^{-}$are almost identical to those from the numerical HNC results for concentrations $x_{2} \leq 10^{-4}$. Thus, numerically, the limiting behavior predicted by Eqs. (18) and (19) pertains to the HNC as well as to the RPA. Note that the coefficients of the leading order terms in Eq. (19) are consistent with the requirement of $\left(A_{12}^{-}\right)^{2}=A_{11}^{-} A_{22}^{-}$.

It is not immediately obvious why the two (very) different closure approximations should yield the same limiting behavior for the pair-correlation functions as $x_{2} \rightarrow 0$. That they do suggests that the limiting behavior described by Eqs. (18) and (19) should be valid more generally. In the Appendix we show that these results follow from the natural division, Eq. (15), of the pair direct correlation functions into a Yukawa tail plus a short-ranged contribution $c_{i j}^{\mathrm{sr}}(r)$. Provided the Fourier transforms $\hat{c}_{i j}^{\mathrm{sr}}(q)$ are sufficiently well behaved in the neighborhood of $q=i \lambda$, Eqs. (18) and (19) should be valid for any closure.

\section{PHASE DIAGRAM AND LIFSHITZ LINE FOR $\delta=0.1$}

The results presented in the last section imply that within the RPA and HNC introducing a small degree of nonideality into the pair potentials defining the mixture, i.e., imposing $\delta>0$, leads to a spinodal pole. The latter dictates that paircorrelation functions should decay monotonically as $r \rightarrow \infty$ for all state points, including those far removed from the spinodal, where one might have expected oscillatory decay (within the HNC). In this section we focus on the spinodal itself and the associated fluid-fluid phase separation.

The spinodal is the locus of points in the phase diagram at which the spinodal pole reaches zero. Within the RPA we can determine the spinodal by finding solutions of $\alpha_{0}^{-}=0$, where $\alpha_{0}^{-}$is given by Eq. (10) with the negative sign. This result is plotted in Fig. 4 for $\delta=0.1$ and $T^{*}=1$ (dashed curve). Within the HNC it is not possible to determine the spinodal precisely. For certain densities and concentrations the HNC approximation does not have solutions. However, it is possible to calculate the position of the HNC spinodal pole as a function of $\rho \lambda^{-3}$ at fixed concentration and extrapolate to zero as shown in Fig. 3. These spinodal points, obtained for a number of concentrations, are shown in Fig. 4 (diamonds). The HNC spinodal lies close to that obtained in the RPA.

Using the RPA we are able to write the reduced bulk Helmholtz free energy per particle $\tilde{f}$ as a sum of ideal and excess parts, ${ }^{4-7}$

$$
\widetilde{f}\left(\rho, x_{2}\right)=\widetilde{f}_{\text {id }}+\frac{1}{2} \rho\left[x_{1}^{2} \hat{\phi}_{11}(0)+2 x_{1} x_{2} \hat{\phi}_{12}(0)+x_{2}^{2} \hat{\phi}_{22}(0)\right],
$$

where $\hat{\phi}_{i j}(0)=4 \pi \epsilon M_{i j} \lambda^{-3}$ is the $q=0$ limit of the Fouriertransformed pair potential. The ideal part $\widetilde{f}_{\text {id }}\left(\rho, x_{2}\right)$ contains the ideal free energy of mixing $\beta^{-1}\left\{x_{1} \ln \left(x_{1}\right)+x_{2} \ln \left(x_{2}\right)\right\}$, as well as a term in $\rho$ that is irrelevant for phase behavior. Equation (20) corresponds to calculating $\tilde{f}$ from the compressibility route, and the spinodal obtained from this equation is identical to that obtained from the zeros of $\alpha_{0}^{-}$. We now Legendre transform to the Gibbs free energy $g=\tilde{f}+P v$, where $v=1 / \rho$ is the volume per particle and the pressure is given by $P=-(\partial \tilde{f} / \partial v)_{x_{2}}$. Using the common tangent construction on $g$ we obtain the binodal which is also plotted in 
Fig. 4 (solid curve). The binodal and spinodal meet at the critical point near $x_{2}=0.27$ and $\rho \lambda^{-3}=0.88$.

In implementing the $\mathrm{HNC}$ we choose to calculate the thermodynamic functions locally, thereby avoiding thermodynamic integration. We begin by tracing out isobars across the phase diagram with the pressure given by the virial route,

$$
\frac{\beta P}{\rho}=1-\frac{2 \pi \rho}{3} \sum_{i} \sum_{j} x_{i} x_{j} \int_{0}^{\infty} d r r^{3} \frac{d \beta \phi_{i j}(r)}{d r} g_{i j}(r) .
$$

Along the isobars we calculate the $\mathrm{HNC}$ chemical potentials,

$$
\begin{aligned}
\beta \mu_{i}= & \ln \left(\rho_{i} \Lambda_{i}^{3}\right) \\
& +\sum_{j} \rho_{j} \int d \mathbf{r}\left\{\frac{h_{i j}(r)}{2}\left[h_{i j}(r)-c_{i j}(r)\right]-c_{i j}(r)\right\},
\end{aligned}
$$

where $\Lambda_{i}$ is the (irrelevant) thermal de Broglie wavelength of species $i$, with $i=1,2$. The Gibbs free energy per particle is then given by $g=x_{1} \mu_{1}+x_{2} \mu_{2}$. By fitting a polynomial to these results we are then able to determine the binodal using the common tangent construction. For the isobars that intersect the region for which the HNC does not provide a solution we calculate the two "branches" of the free energy and construct the common tangent on these; the procedure is equivalent to that used in Ref. 6. Nevertheless, for state points close to the critical point it is not possible to determine the binodal. The HNC binodal is shown in Fig. 4 (crosses connected by tie lines). This lies outside our estimated HNC spinodal and fairly close to the RPA binodal. A slightly better level of agreement between the HNC and RPA results for the binodal was found in Ref. 6 for a soft-core model of binary star polymers.

Since the spinodal pole dictates the ultimate decay of the pair correlations even for states that are far removed from the spinodal it is instructive to seek some criterion which indicates when contributions from the other pole(s) become important in determining the structure of the fluid. One valuable indicator of the change in the latter is the Lifshitz line which focuses on the behavior of the fluid structure at small wave numbers $q{ }^{6,18}$ We define the partial structure factors as $^{19}$

$$
S_{i j}(q)=\delta_{i j}+\left(x_{i} x_{j}\right)^{1 / 2} \rho \hat{h}_{i j}(q),
$$

and concentrate on the number-number structure factor,

$$
S_{\mathrm{NN}}(q)=x_{1} S_{11}(q)+x_{2} S_{22}(q)+2\left(x_{1} x_{2}\right)^{1 / 2} S_{12}(q) .
$$

As we approach the critical point or, indeed, the spinodal, the partial structure factors diverge at small $q: S_{11}(q=0)$ and $S_{22}(q=0) \rightarrow+\infty$ and $S_{12}(q=0) \rightarrow-\infty$. These divergences are reflected in the linear combination, i.e., $S_{\mathrm{NN}}(q=0) \rightarrow+\infty$.

We define the Lifshitz line as separating regions of the phase diagram for which $S_{\mathrm{NN}}(q)$ has a local maximum or a local minimum at $q=0$. We choose $S_{\mathrm{NN}}(q)$ because (i) it is the most symmetrical combination of the partial structure factors, and (ii) it diverges in a similar manner on both sides of the phase diagram. Formally we make a small $q$ expansion of $S_{\mathrm{NN}}(q)$,

$$
S_{\mathrm{NN}}(q)=a\left(\rho, x_{2}\right)+b\left(\rho, x_{2}\right) q^{2}+O\left(q^{4}\right),
$$

so that the Lifshitz line is the locus of points for which $b\left(\rho, x_{2}\right)=0 .{ }^{6,18}$ This line is calculated in both the RPA and HNC and the results shown as the dash-dotted curve and crosses, respectively, in Fig. 4. We find that there is good agreement between the two closures, suggesting that in this region of the phase diagram the RPA should become a fairly reliable approximation for describing $h_{i j}(r)$ at both intermediate and large values of $r$.

\section{DISCUSSION AND CONCLUSIONS}

In this paper we have investigated the structural and thermodynamic properties of binary mixtures of particles interacting via purely repulsive (point) Yukawa pair potentials. We have used two approximate closures to the OZ equations in order to calculate the fluid properties: The simple RPA allows us to elucidate analytically some important properties, and the HNC approximation is expected to be very accurate for the Yukawa fluid at the intermediate fluid densities relevant to the present work. This expectation stems from the fact that the $\mathrm{HNC}$ closure correlation functions are rather accurate for the OCY., ${ }^{9,13}$

For the ideal mixture, with $\delta=0$, the RPA gives a poor description of the fluid structure, predicting monotonic (exponential) decay of $r h_{i j}(r)$ for all state points in the phase diagram rather than the Kirkwood crossover line that is manifested in the HNC-see Fig. 2. No fluid-fluid phase separation occurs for $\delta=0$. By contrast, when $\delta>0$ an additional purely imaginary pole arises within the HNC approximation for $\hat{h}_{i j}(q)$. This spinodal pole governs the phase separation in the mixture for the nonideal case. Since the RPA provides a good account of this pole, see Fig. 3, it follows that the RPA also yields fluid-fluid transition boundaries that are quite close to those from the more accurate HNC-see Fig. 4. The amplitude $A_{i j}^{-}$of the spinodal pole contribution to $h_{i j}(r)$ becomes increasingly large with increasing proximity to the spinodal, and this pole provides the dominant contribution in the region of the phase diagram near the spinodal (roughly the region enclosed by the Lifshitz line-see Fig. 4).

For states away from the spinodal/binodal, particularly in the limits $x_{1} \rightarrow 0$ or $x_{2} \rightarrow 0$, the amplitudes of the contribution from the spinodal pole to the majority species correlation function $h_{i i}(r)$ (where $i$ is the majority species) and to $h_{12}(r)$ become very small—see Figs. 5 and 6. One must magnify the large $r$ tail of these correlation functions in order to see the contribution from the spinodal pole. Above the Kirkwood line, but well away from the spinodal, the intermediate decay of $h_{i j}(r)$ is damped oscillatory; the oscillations can persist up to large $r$, before the monotonic decay from the spinodal pole finally dominates at very large values of $r$. This result is quite unusual, since one generally expects the leading order pole to dominate the decay of $h_{i j}(r)$ at both large and intermediate values of $r \cdot{ }^{9,14,17}$ In the limit $x_{2} \rightarrow 0$, the spinodal pole $\rightarrow i \lambda$ and the amplitudes $A_{11}^{-}$and $A_{12}^{-}$of the contributions of $h_{11}(r)$ and to $h_{12}(r)$ have a power-law dependence on $x_{2}$ [see Eq. (19)], vanishing in the pure fluid of species 1 . However, the amplitude, $A_{22}^{-}$, of the contribution 
from the spinodal pole to $h_{22}(r)$ tends to a nonvanishing value in the limit $x_{2} \rightarrow 0$. This means that for small values of $x_{2}$ the nature of the decay of the correlation function $h_{22}(r)$ can be very different from that of $h_{11}(r)$ and $h_{12}(r)$, a situation which has not been encountered in other fluid mixtures. In experimental or simulation results it would be almost impossible to detect the contribution to $h_{11}(r)$ and $h_{12}(r)$ from the spinodal pole for small $x_{2}$. However, its existence could be inferred from the apparently different decay of $h_{22}(r)$ versus that of $h_{11}(r)$ and $h_{12}(r)$.

It is important to emphasize that the amplitude $A_{22}^{-}$tending to a nonzero value in the limit $x_{2} \rightarrow 0$ does not imply any pathological consequences for thermodynamic or measurable structural quantities. As pointed out earlier, physical observables such as the liquid structure factors involve the product $\rho_{2} h_{22}(r)$ which vanishes as $x_{2} \rightarrow 0$. One exception to this rule is the effective potential between two particles immersed in a solvent of the other species. The effective potential $\phi_{22}^{\text {eff }}(r)$ between two particles of species 2 at infinite dilution is related to the radial distribution function, in the limit $x_{2} \rightarrow 0$, by

$$
g_{22}(r) \equiv \exp \left(-\beta \phi_{22}^{\text {eff }}(r)\right) .
$$

Clearly $\phi_{22}^{\text {eff }}(r)$ depends on the density and temperature of the solvent (species 1). We define the solvent-mediated potential $W_{22}(r)$ via $\phi_{22}^{\text {eff }}(r) \equiv \phi_{22}(r)+W_{22}(r)$, where $\phi_{22}(r)$ is the bare (direct) potential between the two particles. $W_{22}(r)$ depends on the nature of the solvent and on the solvent-particle interaction. From Eq. (26) it follows that $W_{22}(r)$ depends on $h_{22}(r)$ rather than $\rho_{2} h_{22}(r)$. There are clear implications for the form of $\phi_{22}^{\mathrm{eff}}(r)$ given that the amplitude of the contribution to $h_{22}(r)$ from the spinodal pole is nonvanishing in the limit $x_{2} \rightarrow 0$. We leave a full discussion to another publication but point out that the presence of the spinodal pole, which occurs for any $\delta>0$, gives rise to an effective potential that is attractive at sufficiently large distances and decays as $\exp (-\lambda r) / r$, i.e., with the same length scale as the bare potentials.

Given that the Yukawa potential arises in a great variety of physical problems, ${ }^{20}$ typically where there are big charged particles screened by a neutralizing background medium, we believe the present work should be relevant to systems ranging from charged colloidal fluids ${ }^{1}$ to dusty plasmas. ${ }^{2}$

We have chosen to focus on a simple example of a binary Yukawa mixture, namely, one in which the inverse screening length $\lambda$ is the same for all $i j$ pairs, as this is the situation which arises naturally for charged particles immersed in a neutralizing medium. One could, of course, consider more complex examples with $\lambda$ dependent on the species indices $i, j$. Moreover, one could consider mixtures that correspond to negative nonadditivity, i.e., with $\delta<0$. In this case $M_{12}<\sqrt{M_{11} M_{22}}$ and the interactions should favor fluidfluid mixing.

\section{ACKNOWLEDGMENTS}

One of the authors (P.H.) is grateful for the support of an EPSRC studentship and another author (A.J.A.) acknowledges the support of EPSRC under Grant No. GR/S28631/01.

\section{APPENDIX: BEHAVIOR OF CORRELATION FUNCTIONS AS $x_{2} \rightarrow 0$}

Here we show that the results for the spinodal pole and the amplitudes $A_{i j}^{-}$, Eqs. (18) and (19), in the limit $x_{2} \rightarrow 0$ are not specific to the RPA but follow generally from the form of the direct correlation functions for Yukawa mixtures. Making the separation of the $c_{i j}(r)$ given by Eq. (15) and substituting into Eq. (5) we obtain

$$
D(q)=a+\frac{b}{p}+\frac{c}{p^{2}},
$$

where

$$
\begin{aligned}
p= & q^{2}+\lambda^{2}, \\
a= & {\left[1-\rho_{1} c_{11}^{\mathrm{sr}}(q)\right]\left[1-\rho_{2} c_{22}^{\mathrm{sr}}(q)\right]-\rho_{1} \rho_{2}\left[c_{12}^{\mathrm{sr}}(q)\right]^{2}, } \\
b= & {\left[1-\rho_{1} c_{11}^{\mathrm{sr}}(q)\right] \rho_{2} \alpha_{22}+\left[1-\rho_{2} c_{22}^{\mathrm{sr}}(q)\right] \rho_{1} \alpha_{11} } \\
& +2 \rho_{1} \rho_{2} c_{12}^{\mathrm{sr}}(q) \alpha_{12}, \\
c= & \rho_{1} \rho_{2}\left(\alpha_{11} \alpha_{22}-\alpha_{12}^{2}\right),
\end{aligned}
$$

and $\alpha_{i j}=4 \pi M_{i j} / \lambda T^{*}$. The poles are given by the solution to the equation $D(q)=0$. From Eq. (A1) we see that one set of solutions is given by

$$
p_{ \pm}=-\left(b \pm \sqrt{b^{2}-4 a c}\right) / 2 a .
$$

$a$ and $b$ are functions of $q$. However, Eq. (A3) leads to purely imaginary poles at $q_{ \pm}=i \alpha_{0}=i \sqrt{\lambda^{2}-p_{ \pm}}$, provided we assume that the functions $c_{i j}^{\mathrm{sr}}(q)$ are well behaved (finite and differentiable) on the imaginary axis around $q_{ \pm}$. The leading order pole is that corresponding to $p_{-}$, which in the limit of vanishing density $\rho_{2}=0$ (i.e., $c=0$ ) yields a pole at $q=i \lambda$, giving a decay $r h_{i j}(r) \sim A_{i j}^{-} \exp (-\lambda r)$, where the amplitudes $A_{i j}^{-}$are to be determined below. For small concentrations of species 2 we can Taylor expand $p_{-}$in powers of $c$, giving

$$
p_{-}=-\frac{c}{\rho_{1} \alpha_{11}}+\left(\frac{c\left(b-\rho_{1} \alpha_{11}\right)}{\rho_{1}^{2} \alpha_{11}^{2}}-\frac{a c^{2}}{\rho_{1}^{3} \alpha_{11}^{3}}\right)+O\left(\rho_{2}^{3}\right) \text {. }
$$

We find that in the limit $\rho_{2} \rightarrow 0, p_{-} \sim-\left(\alpha_{22}-\alpha_{12}^{2} / \alpha_{11}\right) \rho_{2}$. Thus the leading order pole has

$$
\alpha_{0}^{-}=\lambda\left(1-\frac{\alpha_{11} \alpha_{22}-\alpha_{12}^{2}}{2 \lambda^{2} \alpha_{11}} \rho_{2}\right)+O\left(\rho_{2}^{2}\right),
$$

which is identical to the RPA result in Eq. (18).

The amplitude $A_{i j}^{-}$is given by

$$
A_{i j}^{-}=\frac{q_{-} N_{i j}\left(q_{-}\right)}{2 \pi D^{\prime}\left(q_{-}\right)},
$$

where the prime denotes the derivative with respect to $q$ and the functions $N_{i j}(q)$ are given in Eq. (6). From Eq. (A1) we find that

$$
D^{\prime}(q)=a^{\prime}+\frac{b^{\prime}}{p}-\frac{2 q b}{p^{2}}-\frac{4 q c}{p^{3}} .
$$

In the limit $\rho_{2} \rightarrow 0$, using (A4), we find that $D^{\prime}\left(q_{-}\right)$ $=2 q_{-} b / p_{-}^{2}+$ other terms less singular in $\rho_{2}$. Using this result, we can expand Eq. (A6) to obtain the amplitudes $A_{i j}^{-}$in the 
limit $\rho_{2} \rightarrow 0$. We find that the leading order terms are precisely the RPA results given in Eq. (19), i.e., in the limit $\rho_{2}$ $\rightarrow 0$ the amplitudes $A_{i j}^{-}$are independent of the functions $\hat{c}_{i j}^{\mathrm{sr}}(q)$.

${ }^{1}$ See, e.g., J.-P. Hansen and H. Löwen, Annu. Rev. Phys. Chem. 51, 209 (2000), and references therein.

${ }^{2}$ For a recent review see A. Piel and A. Melzer, Adv. Space Res. 29, 1255 (2002), and references therein.

${ }^{3}$ J.-P. Hansen and I. R. McDonald, Theory of Simple Liquids, 2nd ed. (Academic, London, 1986).

${ }^{4}$ A. A. Louis, P. G. Bolhuis, and J.-P. Hansen, Phys. Rev. E 62, 7961 (2000).

${ }^{5}$ A. J. Archer and R. Evans, Phys. Rev. E 64, 041501 (2001).

${ }^{6}$ A. J. Archer, C. N. Likos, and R. Evans, J. Phys.: Condens. Matter 14, 12031 (2002).

${ }^{7}$ R. Finken, J.-P. Hansen, and A. A. Louis, J. Stat. Phys. 110, 1015 (2003).

${ }^{8}$ M. Baus and J.-P. Hansen, Phys. Rep. 59, 1 (1980), and references therein.

${ }^{9}$ P. Hopkins, A. J. Archer, and R. Evans, Phys. Rev. E 71, 027401 (2005).

${ }^{10}$ C. N. Likos, Phys. Rep. 348, 267 (2001).
${ }^{11}$ C. N. Likos, A. Lang, M. Watzlawek, and H. Löwen, Phys. Rev. E 63, 031206 (2001).

${ }^{12}$ A. Lang, C. N. Likos, M. Watzlawek, and H Löwen, J. Phys.: Condens. Matter 12, 5087 (2000).

${ }^{13}$ W. Daughton, M. S. Murillo, and L. Thode, Phys. Rev. E 61, 2129 (2000).

${ }^{14}$ R. Evans, R. J. F Leote de Carvalho, J. R. Henderson, and D. C. Hoyle, J. Chem. Phys. 100, 591 (1994); see also G. A. Martynov, Fundamental Theory of Liquids: Methods of Distribution Functions (Hilger, Bristol, 1992).

${ }^{15}$ Note that the RPA results of Eqs. (11) and (13) predict unphysical behavior of $h_{i j}(r)$ as $r \rightarrow 0$. However, we are concerned here with $h_{i j}(r)$ as $r \rightarrow \infty$ where the RPA results do capture the essential physics.

${ }^{16}$ J. G. Kirkwood, Chem. Rev. (Washington, D.C.) 19, 275 (1936).

${ }^{17}$ R. J. F Leote de Carvalho, R. Evans, and Y. Rosenfeld, Phys. Rev. E 59, 1435 (1999)

${ }^{18}$ G. Gompper and M. Schick, Phys. Rev. B 41, 9148 (1990); R. M. Hornreich, R. Liebmann, H. G. Schuster, and W. Selke, Z. Phys. B 35, 91 (1979).

${ }^{19}$ See, e.g., C. Caccamo, Phys. Rep. 274, 1 (1996), and references therein.

${ }^{20}$ J. S. Rowlinson, Physica A 156, 15 (1989). 